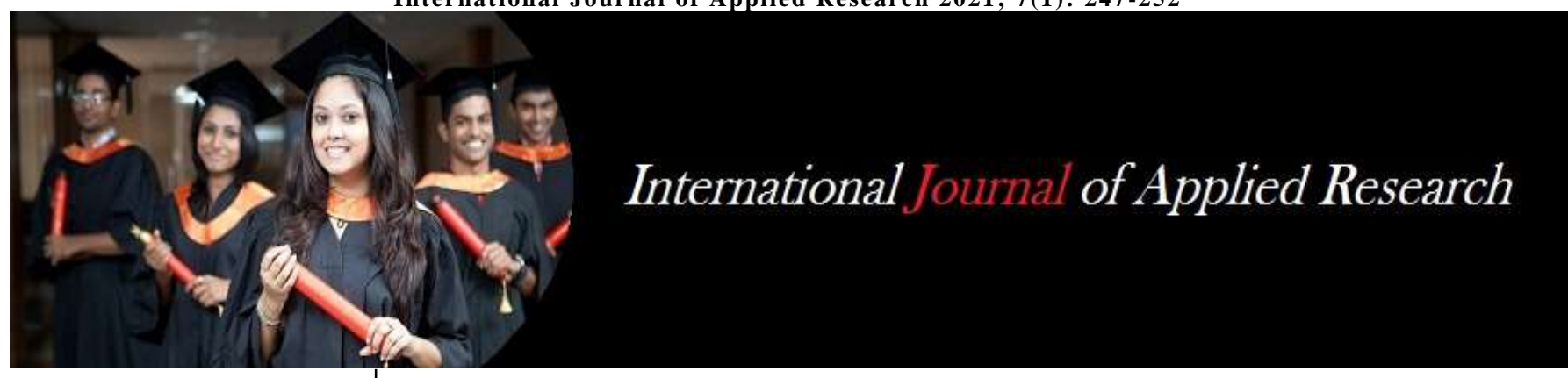

ISSN Print: 2394-7500 ISSN Online: 2394-5869 Impact Factor: 8.4

IJAR 2021; 7(1): 247-252 www.allresearchjournal.com

Received: 02-11-2020 Accepted: 11-12-2020

\section{कल्पना कुमारी}

गृह विज्ञान विभाग, राँची विश्वविधालय, राँची, झारखंड, भारत

\section{डॉ सीमा डे}

गह विज्ञान विभाग, राँची विश्वविधालय, राँची, झारखंड, भारत

Corresponding Author: कल्पना कुमारी गह विज्ञान विभाग, राँची विश्वविधालय, राँची, झारखंड, भारत

\section{बाल-श्रमिकों की सामाजिक, आर्थिक स्थिति का अध्ययन : "राँची जिला के संदर्भ में"}

\section{कल्पना कुमारी एवं डॉ सीमा डे}

DOI: $\underline{\text { https://doi.org/10.22271/allresearch.2021.v7.i1d.8186 }}$

\section{सारांश}

भारत में बाल-श्रम समस्या एक चुनौती बन गई है। देश में कामकाजी बच्चों की वास्तविक संख्या बहुत ज्यादा है | जातिवाद, गरीबी, परिवार का आकार, तथा आय, शिक्षा का स्तर आदि बाल-श्रमिक को गम्भीर समस्या के रूप में प्रकट करने के लिए उत्तरदायी है | बाल- श्रम समस्या एक गहन सामाजिकआर्थिक समस्या है। यह एक ऐसी बुराई है जिसके लिए समाज के सभी वर्गों में जागरूकता लाने के साथ-साथ सोच का नजरिया भी बदलने की जरुरत है। बाल मजदूरी करने वाले अधिकांश बच्चे पिछड़ी जातियों और पिछड़ों में भी अति पिछड़े वर्ग से आते हैं। इसका एक बड़ा कारण चेतना की कमी है | बच्चे देश के भविष्य होते हैं।

बाल-श्रम न सिर्फ बच्चों के लिए अभिशाप के समान है बल्कि यह समाज और देश के माथे पर कलंक है। भारत में बाल-श्रमिक समस्या अत्यन्त जटिल है लेकिन यह सिर्फ भारत की समस्या नहीं बल्कि एक अन्तर्राष्ट्रीय परिघटना है। इस शोध अध्ययन में राँची जिला से 100 बाल-मजदूरों को रैंडम रूप से चयनित किया गया था। इस अध्ययन में बाल-मजदूरों के व्यक्तिगत, गुण स्वतन्त्र चर है तथा अधिकांश 50 प्रतिशत बालिका तथा 40 प्रतिशत बालक 13-14 वर्ष के पाये गये उनकी सामाजिक, आर्थिक स्थिति आश्रित चर हैं। अधिकांश 56 प्रतिशत बालिका तथा 50 प्रतिशत बालक निरक्षर थे जबकि 10 प्रतिशत बालिका तथा 4 प्रतिशत बालक हाईस्कूल तक शिक्षा प्राप्त की थी। उत्तरदाताओं में 38 प्रतिशत बालक पिछड़ी जाति के थे जबकि 44 प्रतिशत बालिका अनुसूचित जनजाति की थी। अधिकांश 70 प्रतिशत बालक तथा 64 प्रतिशत बालिका एकल परिवार में रहते थे तथा उनके परिवार के स्वरूप में छ: या इससे अधिक सदस्यों वाले 60 प्रतिशत बालक तथा 64 प्रतिशत बालिका थी। अधिकांश लोग कच्चे मकान में रहते थे। पानी के साधन के रूप में वे कुआँ का प्रयोग करते थे। 74 प्रतिशत बालक 80 प्रतिशत बालिका को माता-पिता के साथ थोड़ा ही रहने का समय मिल पाता था। बच्चों के काम करने से उनकी पारिवारिक आय में वृद्धि हुई थी। अधिकत्तर लोग दैनिक आहार में केवल चावल सब्जी का ही प्रयोग करते थे। अधिकांश बालक तथा बालिका रेजा का काम कर अपने गुजारा करते थे। इनके परिवार के आय का मुख्य स्तोत मजदूरी ही था तथा इनके परिवार के मासिक आय 1501-4500 से नीचे थी। इनके दैनिक मजदूरी का आधार में 28 प्रतिशत बालक तथा 22 प्रतिशत बालिका को मालिक की इच्छा के अनुसार मजदूरी मिलती थी।

प्रमुख शब्द : बाल श्रमिक, पारिवारिक, सामाजिक, आर्थिक

\section{परिचय}

हमारे देश का भविष्य बच्चों में निहित है । ये देश के कर्णधार और अपने परिवार की धरोहर है | सम्पर्ण मानव समाज के लिए कलंक बनी यह समस्या अपना विकट रूप धारण कर रही है । यह प्राचीन काल से ही चला आ रहा है । यह बच्चों के स्वास्थ्य को भी प्रभावित करती है तथा शारीरिक एवं मानसिक विकास में बाधा पहुँचाती है। बच्चे राष्ट्र के भावी कर्णधार होते है, बच्चे राष्ट्र के भविष्य है, 
आज का बच्चा कल का नागरिक है, फिर भी आज भारत के विभित्र क्षेत्रों में लाखों बच्चे शोषण के शिकार हो रहे है| चौदह वर्ष से कम आयु के मानसिक व शारीरिक श्रम करने वाले बच्चे को बाल श्रमिक कहा जाता है | गाँवों से लेकर शहरों तक, असंगठित दुकानों एवं प्रतिष्ठानों से लेकर कुछ संगठित कारखानों तक में लम्बे अरसे से बाल—श्रमिक काम करते हैं। जिन बच्चों के हाथों में खिलौने, कागज-कलम, कॉपी-किताब, स्लेट—पेन्सिल होनी चाहिए थी, उन हाथों में औरों के जूते पॉलिश करने के ब्रश, दूसरों के पढ़ने के लिए स्लेट-निर्माण की सामग्रियाँ, पत्थर तोड़ने के हथौड़े अथवा दरी—कालीन बुनने के लिए धागों का जाल होता है जिसके मकड़जाल में उनकी जिन्दगी पिसती रहती है | [शर्मा 2010]

देश में करोड़ों की संख्या में गाँव और शहर में बालिका मजदूर भी कई खतरनाक तथा गैर खतरनाक कार्यों में लगी है। इन बालिकाओं की मजदूरी सबसे कम मेहनताना पाने वाले बाल मजदूरों ' से भी कम है। सुबह 6 बजे से शाम के 8 बजे तक कार्य करना सामान्य-सी बात है, कार्य स्थल भी इनके लिए सुरक्षित नहीं है। इनके ऊपर सदैव यौन शोषण की आशंका की तलवार लटकती रहती है, भारत काफी पूर्व से, आज तक कृषि प्रधान देश रहा है। भारत के विभित्र प्रान्तो में कृषि मजदूर एवं कृषि से जुड़े अन्ये सभी कार्यों में बालिकाओ की सहभागिता है / कुछ क्षेत्रों में तो बालिकाएँ सर्वाधिक सलग्र है। भारतीय अर्थव्यवस्था की एक महत्वपूर्ण विशेषता गाँव में रहने वाली एक बहुत बड़ी जनसंख्या है, जो मुख्यत: कृषि एवं उससे जुड़े अन्य कार्य पर आश्रित है | (सिंह 2011)

बाल-मजदूरी प्रथा का मुख्य कारण गरीबी, अशिक्षा, वयस्कों की बेरोजगारी तथा कम मजदूरी-दर है। बाल-मजदूरी प्रथा का उन्मूलन सिर्फ सरकारी कार्यक्रमों से नहीं हो सकता। इसके लिए समाज के सभी तबकों यथा स्वयंसेवी संस्थाओं, बुद्धिजीवियों, पत्रकारों, श्रम संघों, सामाजिक कार्यकर्त्ताओं, शिक्षकों, सरकारी कर्मचारियों आदि को एकजुट होकर प्रयास करना होगा | (शर्मा 2010)

\section{अध्ययन का उद्धेश्य}

- बाल श्रमिकों की सामाजिक एवं आर्थिक स्थिति का अध्ययन करना।

\section{परिकल्पना}

(i) अशिक्षा एवं निर्धनता के कारण माता-पिता अपने बच्चों को सही राह दिखाने में असमर्थ होते है तथा अच्छे संस्कार भी नहीं दे पाते है। (ii) परिवार का बालक पर अनेक प्रकार से प्रभाव पड़ता है | जैसे परिवार का टूटना, माता-पिता का तलाक, माता-पिता दोनों में से किसी एक के या दोनो की मृत्यु होने से या किसी के कारावास होना या बाहर होना आदि। इन सबों के कारण वे आर्थिक रूप से कमजोर भी होते हैं।

\section{अध्ययन का महत्त्व}

बच्चों को भावी कर्णधार और आने वाले कल की तस्वीर कहा जाता है। सरकार ने भी बच्चों को राष्ट्र की महत्वपूर्ण संपत्ति स्वीकार किया है। लेकिन कल के उज्वल भविष्य का वर्तमान दो रोटी की लड़ाई में ही इस कदर उलझा है, कि उसके सारे अधिकार बेईमानी साबित हो रहे है। आने वाले कल के कर्णधारों का एक बहुत बड़ा हिस्सा भूख, कुपोषित, शोषित, अशिक्षित, अधिकारों से वंचित और उत्पीड़ित है। बाल अधिकार और बचपन बचाऔं'महज नारा बनकर रह गए है। केंद्र और राज्य सरकारें इसके प्रति उदासीन हैं, जिसका खामियाजा बच्चों को उठाना पड़ रहा है। बाल मजदूरों के प्रमुख समस्याओं के निदान के लिए उसके अनुरूप कार्यक्रम बनाकर प्रशिक्षण दिया जा सकता है जिससे उनकी सामाजिक तथा आर्थिक स्थिति में सुधार हो सके।

\section{अध्ययन की सीमाएं}

शोध अध्ययन के लिए राँची के शहरी क्षेत्र (बिरसा चौक) से 100 बाल मजदूरों का ही चयन किया गया है।

\section{पूर्व अध्ययनों की समीक्षा}

खांडेकर, (1970) के अनुसार 81 प्रतिशत बच्चें वृहत मुम्बई मे कार्यरत हैं। लड़कियाँ तो बाल वेश्यावृति की माँग है, एवं कुछ घरेलू नौकरी में | आर्थिक परिस्थितियाँ निर्धन परिवार की बालिकाओं को मजदूर बनाने एवं बाल वेश्यावृति जैसे घृणित व्यवसायों को करने के लिए बाध्य करती है।

गुलाटी (1981) के अनुसार नारियल उधोग में बालक एवं बालिकाओं से अलग-अलग कार्य कराया जाता है कपड़ों में सिले हुये वस्त्रों में हुक लगाने का कार्य लड़कियों का है। इस उधोग में हर उम्र की लड़कियाँ कार्य करते हुये पायी गयी, जबकि लड़के 9 से 11 वर्ष तक के ही संलग्र पाये गये थे।

अवस्थी (1986) के अनुसार बोगस इकाइयों के मालिक अपने कोटे का कोयला चोर बाजारी में बेच कर 10 लाख रुपये की सालाना आमदनी कमा सकते है, इन इकाइयों में लगभग 50 हजार बाल - श्रमिक कम करते हैं तथा कुल श्रमिकों की संख्या दो लाख है। ये बाल श्रमिक 800 डिग्री सेल्सियस तक के तापमान वाली भट्ठियों में काम करते है। 
अवाचट (1988) ने अपने अध्ययन में पाया कि इचालंकरंजी (महाराष्ट्र) के एक पॉवरलूम फैक्ट्री में 13 वर्ष की एक बच्ची घायल हो गई और अन्तत: उसका एक हाथ काटना पड़ा। किन्तु उसे मुआवजा नहीं मिला क्योंकि उस कारखाने के मालिक ने कहा कि वह लड़की मस्टर रोल पर नहीं है, इसलिए मुआवजा देने का प्रश्न ही नहीं उठता हैं।

खान (1993) ने पाया मिर्जापुर-भदोही क्षेत्र में करीब ढाई लाख बाल - श्रमिक कालीन उधोग में कार्यरत हैं जो पूरे देश द्वारा निर्यात किए जाने वाले कालीनों का 80 प्रतिशत निर्यात करता है।

\section{शोध विधि}

इस शोध अध्ययन का क्षेत्र राँची जिला का बिरसा चौक सिंह मोड़, हटियाऔर टुपू दाना है / प्रत्येक शोध क्षेत्र से 25-25 बाल मजदूरों को रैण्डम रूप से चयनित किया गया प्रतिदर्शियों की कुल संख्या 100 थी / प्रस्ताविक अनुसंधान में प्राथमिक स्त्रोत से सूचनाएँ संकलित की गयी | उद्देश्य से सम्बंधित प्रश्नावली तैयार किया गया तथा आंकड़ो का संकलन किया गया। इसके बाद सारणी बनाया गया तथा निष्कर्ष निकाला गया।

\section{आंकड़ो का सारणीयन एवं विश्लेषण}

सारणी - 1: बाल श्रमिकों की पारिवारिक स्थिति

\begin{tabular}{|c|c|c|c|c|}
\hline \multirow{2}{*}{ आयु } & \multicolumn{2}{|c|}{ बालक } & \multicolumn{2}{c|}{ बालिका } \\
\cline { 2 - 5 } संख्या प्रतिशत & संख्या & प्रतिशत \\
\hline $7-8$ & 5 & 10 & 3 & 6 \\
\hline $9-10$ & 10 & 20 & 12 & 24 \\
\hline $11-12$ & 15 & 30 & 10 & 20 \\
\hline $13-14$ & 20 & 40 & 25 & 50 \\
\hline \multicolumn{5}{|c|}{ शिक्षा की स्थिति } \\
\hline निरक्षर & 25 & 50 & 28 & 56 \\
\hline प्राईमरी & 13 & 26 & 2 & 4 \\
\hline मिडिल & 10 & 20 & 15 & 30 \\
\hline हाईस्कूल & 2 & 4 & 5 & 10 \\
\hline \multicolumn{5}{|c|}{ जाति/समुदाय } \\
\hline उच्च जाति & 3 & 6 & 2 & 4 \\
\hline पिछड़ीजाति & 19 & 38 & 16 & 32 \\
\hline अनुसूचित जाति & 12 & 24 & 15 & 30 \\
\hline अनुसूचित जनजाति & 16 & 32 & 17 & 34 \\
\hline \multicolumn{5}{|c|}{ परिवार का प्रकार } \\
\hline एकल & 35 & 70 & 32 & 64 \\
\hline संयुक्त & 15 & 30 & 18 & 36 \\
\hline परिवार के आकार \\
\hline 2 से 5 सदस्य & 20 & 40 & 18 & 36 \\
\hline 6 या इससे अधिक सदस्य & 30 & 60 & 32 & 64 \\
\hline
\end{tabular}

उपरोक्त सारणी : 1 से ज्ञात होता है कि 40 प्रतिशत बालक तथा 50 प्रतिशत बालिका 13-14 आयु वर्ग के थे। गरीबी एवं बड़े परिवारों के कारण निम्न आय वर्ग के माता पिता स्वयं की आय के स्रोत द्वारा परिवार का भरण-पोषण करने में असमर्थ होते हैं इसी कारण बच्चे 7 से 8 वर्ष की आयु में ही इस कुप्रथा का शिकार हो जाते हैं। इससे पता चलता है कि इतने कम आयु में ही ये बच्चें बाल श्रमिक बन गए है जबकि यह अवस्था उनके खेलने कूदने और विद्धाअर्जन करने की है | बच्चे के व्यक्तित्व के निर्माण में सबसे अहम भूमिका शिक्षा की होती है। शिक्षा और इसकी उपलब्धता की स्थिति
कम उम्र में बच्चों को काम करने को उन्मुख करती है । बच्चों में साक्षरता का स्तर अलग-अलग पाया गया। इसमें कुछ अशिक्षित तथा कुछ शिक्षित हैं और कुछ हाई स्कूल तक की शिक्षा प्राप्त की थी। अधिकांश 50 प्रतिशत बालक और 56 प्रतिशत बालिका निरक्षर थे। जबकि 26 प्रतिशत बालक और 4 प्रतिशत बालिका प्राईमरी और 4 प्रतिशत बालक और 10 प्रतिशत बालिका हाईस्कूल तक की ही शिक्षा ग्रहण की थी। ग्रामीण समाज में खासकर निरक्षर समुदाय में रूढ़िवादिता है जहाँ बालिकाओं की शिक्षा पर ध्यान नहीं दिया जाता है। 32 प्रतिशत बालक और 34 
प्रतिशत बालिका अनुसूचित जनजाति परिवार के थे जबकि 38 बालक 32 प्रतिशत बालिका पिछड़ी जाति के थे। एकल परिवार में रहने वाले 70 प्रतिशत बालक तथा 64 प्रतिशत बालिका थी जबकि 30 प्रतिशत बालक और 36 प्रतिशत बालिका संयुक्त परिवार में रहते थे। संयुक्त परिवार में बच्चों के पालन पोषण में अधिक कठिनाई नहीं आती है जितनी की एकल परिवार में आती है | अधिकांश 60 प्रतिशत बालक तथा
64 प्रतिशत बालिका के परिवार में सदस्यों की संख्या 6 से अधिक की थी जबकि 40 प्रतिशत बालक तथा 36 प्रतिशत बालिका के परिवार में सदस्यों की संख्या 2 से 5 थी। गरीबी व परिवार में सदस्यों की संख्या अधिक होने के कारण बालकों को भी मजदूरी पर लगाना अभिभावकों की विवशता बन जाती है ताकि परिवार का गुजारा हो जाये।

सारणी -2: बाल श्रमिकों की सामाजिक स्थिति

\begin{tabular}{|c|c|c|c|c|}
\hline \multirow{2}{*}{ घर } & \multicolumn{2}{|c|}{ बालक } & \multicolumn{2}{|c|}{ बालिका } \\
\hline & संख्या & प्रतिशत & संख्या & प्रतिशत \\
\hline कच्चा मकान & 35 & 70 & 41 & 82 \\
\hline पक्का मकान & 15 & 30 & 9 & 18 \\
\hline \multicolumn{5}{|c|}{ पानी के स्त्रोत } \\
\hline कुआँ & 35 & 70 & 28 & 56 \\
\hline तालाब & 2 & 4 & 4 & 8 \\
\hline चापानल & 13 & 26 & 18 & 36 \\
\hline नदी & 00 & 00 & 00 & 00 \\
\hline \multicolumn{5}{|c|}{ माता—पिता के साथ समय } \\
\hline थोड़ा & 37 & 74 & 40 & 80 \\
\hline बहुत & 3 & 6 & 7 & 14 \\
\hline बिलकुल नहीं & 10 & 20 & 13 & 26 \\
\hline \multicolumn{5}{|c|}{ काम करने की प्रेरणा } \\
\hline परिवार & 27 & 54 & 22 & 44 \\
\hline पड़ोस & 11 & 22 & 17 & 34 \\
\hline अन्य & 12 & 24 & 11 & 22 \\
\hline \multicolumn{5}{|c|}{ परिवार पर प्रभाव } \\
\hline पारिवारिक आय में वृद्धि & 46 & 92 & 42 & 84 \\
\hline सामाजिक प्रतिष्ठा में वृद्धि & 4 & 8 & 8 & 16 \\
\hline \multicolumn{5}{|c|}{ भोजन की स्थिति } \\
\hline चावल दाल सब्जी & 13 & 26 & 14 & 28 \\
\hline केवल चावल सब्जी & 18 & 36 & 17 & 34 \\
\hline रोटी सब्जी & 12 & 24 & 11 & 22 \\
\hline मांस—मछली & 7 & 14 & 8 & 16 \\
\hline
\end{tabular}

उपरोक्त सारणी -2 से ज्ञात होता है कि अधिकांश 70 प्रतिशत बालक और 82 प्रतिशत बालिका कच्चे मकान में रहते थे जबकि 30 प्रतिशत बालक और 18 प्रतिशत बालिका पक्का मकान मे रहते थे। अधिकांश 70 प्रतिशत बालक और 56 प्रतिशत बालिका कुआँ का पानी पीते थे, जबकि 4 प्रतिशत बालक और 8 प्रतिशत बालिका तथा 26 प्रतिशत बालक और 36 प्रतिशत बालिका चापानल का पानी इस्तेमाल करते थे। अधिकांश बालक तथा बालिकाओं का कहना था कि उनके माता-पिता काम करने के कारण थोड़ा ही समय दे पाते है, जबकि 6 प्रतिशत बालक और 14
प्रतिशत बालिकाओं ने कहा है कि उनके परिवार वाले उनकों पूरा समय देते थे क्योंकि वे घर से ही अपना काम करती थी बीस प्रतिशत बालक बालक तथा 26 प्रतिशत बालिका ने बताया कि उनके माता-पिता बिलकुल ही उनके साथ समय व्यतीत नहीं करते थे। 54 प्रतिशत बालक और 44 प्रतिशत बालिका ने कहा कि वर्तमान कार्य अपने परिवार की प्रेरणा लेकर कर रहे थे जबकि 24 प्रतिशत बालक और 22 प्रतिशत बालिका ने यह स्वीकार किया कि वे वर्तमान काम अन्य दोस्त व नातेदार की प्रेरणा से कर रहे थे। अधिकांश 92 प्रतिशत बालक और 84 प्रतिशत बालिका का 
मानना था कि उनके उधोग में काम करने से उनके पारिवारिक आय में वृद्धि हुई है जबकि 8 प्रतिशत बालक 16 प्रतिशत बालिका ने कहा है कि उधोग में काम करने से परिवार के सदस्यों का सामाजिक प्रतिष्ठा में वृद्धि हुई है | अधिकत्तर 36 प्रतिशत बालक और 34
प्रतिशत बालिका ने कहा है कि वे केवल चावल सब्जी खाकर ही अपना गुजरा करते थे। जबकि 14 प्रतिशत बालक और 16 प्रतिशत बालिका का मानना था कि वे कभी-कभी मांस-मछली भी खाते थे।

सारणी -3: बाल श्रमिकों की आर्थिक स्थिति

\begin{tabular}{|c|c|c|c|c|}
\hline \multirow{2}{*}{ पेशा } & \multicolumn{2}{|c|}{ बालक } & \multicolumn{2}{|c|}{ बालिका } \\
\hline & संख्या & प्रतिशत & संख्या & प्रतिशत \\
\hline खेत में काम करने वाले & 5 & 10 & 2 & 4 \\
\hline रेज़ा का काम & 20 & 40 & 23 & 46 \\
\hline घर का काम & 3 & 6 & 15 & 30 \\
\hline अन्य & 22 & 44 & 10 & 20 \\
\hline \multicolumn{5}{|c|}{ कार्य करने की अवधि } \\
\hline $2-4$ & 12 & 24 & 10 & 20 \\
\hline $5-7$ & 25 & 50 & 28 & 56 \\
\hline $7-10$ & 13 & 26 & 12 & 24 \\
\hline \multicolumn{5}{|c|}{ परिवार के आय के स्त्रोत } \\
\hline नौकरी & 12 & 24 & 10 & 20 \\
\hline व्यापार & 14 & 28 & 12 & 24 \\
\hline मजदूरी & 25 & 50 & 30 & 60 \\
\hline दूकानदारी & 10 & 20 & 13 & 26 \\
\hline कृषि & 23 & 46 & 24 & 48 \\
\hline पशुपालन & 20 & 40 & 29 & 58 \\
\hline बेरोजगारी & 12 & 24 & 9 & 18 \\
\hline गृहणी & 00 & 00 & 7 & 14 \\
\hline \multicolumn{5}{|c|}{ मासिक आय } \\
\hline 1500 रु से नीचे & 10 & 20 & 15 & 30 \\
\hline 1501 से 4500 & 30 & 60 & 28 & 56 \\
\hline 4501 से 7500 & 7 & 14 & 5 & 10 \\
\hline 7501 से उपर & 3 & 6 & 2 & 4 \\
\hline \multicolumn{5}{|c|}{ मजदूरी के आधार } \\
\hline प्रतिदिन & 20 & 40 & 28 & 56 \\
\hline सप्ताह में & 4 & 8 & 2 & 4 \\
\hline महीने में एक बार & 12 & 24 & 9 & 18 \\
\hline मालिक की इच्छा से & 14 & 28 & 11 & 22 \\
\hline
\end{tabular}

उपरोक्त सारणी 3 से ज्ञात होता है कि रेज़ा का काम करने वाले 40 प्रतिशत बालक और बालिका 46 प्रतिशत थे जबकि घर का काम करने वाले 6 प्रतिशत बालक और 30 प्रतिशत बालिका, जबकि खेत का काम करने वाले 10 प्रतिशत बालक और 4 प्रतिशत बालिका थी। 50 प्रतिशत बालक 56 प्रतिशत बालिका थी पाँच से सात साल से काम करने वाले जबकि 24 प्रतिशत बालक और 20 प्रतिशत बालिका 2-4 साल से काम कर रहे थे | पचास प्रतिशत पिता और 60 प्रतिशत माता मजदूरी करते थे, जबकि 46 प्रतिशत पिता और 48
प्रतिशत माता कृषि कार्य में संलग्र थी। 60 प्रतिशत बालक और 56 प्रतिशत बालिका के परिवार की आय 1501 से 4500 रू तक थे जबकि 6 प्रतिशत बालक और 4 प्रतिशत बालिका के परिवार की आय 7500 रू से उपर थे | परिवार का कम मासिक आय बाल मजदूर होने का एक बहुत बड़ा कारण है। निम्न आर्थिक स्थिति के कारण इनकी स्वास्थ्य संबंधी स्थिति दयनीय है, जो कुपोषण का एक बहुत बड़ा कारण है | चालीस प्रतिशत बालक और 56 प्रतिशत बालिकाओं को दैनिक मजदूरी प्रतिदिन शाम में मिलते थे, 8 प्रतिशत बालक और 4 
प्रतिशत बालिकाओं को दैनिक मजदूरी सप्ताह में और 24 प्रतिशत बालक और 18 प्रतिशत बालिका को दैनिक मजदूरी महीने में एक बार मिलते थे।

\section{निष्कर्ष :}

शोध में पाया कि लगभग शत-प्रतिशत बाल श्रमिक शिक्षा के प्रति जागरूक थे। लगभग प्रत्येक जाति और समुदाय के बाल श्रमिकों को शिक्षा का महत्त्व पता थे। तथा वे अपनेपर दुखी थे क्योंकि वे शिक्षित नहीं है, तथा वे अपने आप को शिक्षित करना चाहते थे। मगर गरीबी के कारण वे मजदूरी करने को विवश हो जाते है। वे वर्तमान समाज में शिक्षा एवं नौकरी के महत्व को जानते है । उन्हें पता है कि बिना नौकरी के मजदूरी तथा उससे संबंधित कार्यों से उन्हें इतनी आय प्राप्त नहीं होती है जिससे उनका जीवन सुखी हो, उनकी जरूरतें पूरी हों सके । यहाँ तक कि मजदूरी कार्य से प्राप्त आय से उनकी न्यूनतम आवश्यकताए भी पूरीनहीं हो पाते है | औधोगिकरण एवं शहरीकरण के कारण बाल-श्रमिकों का रुख दिन-प्रतिदिन शहरों की तरफ जा रहा है। शिक्षा और इसकी उपलब्धता की स्थिति भी कम उम्र में बच्चों को काम करने को प्रेरित करती है । यह एक ऐसी सामाजिक बुराई है जिसके लिए समाज के सभी वर्गों में जागरूकता के प्रसार तथा एक परिवर्तित दृष्टिकोण की आवश्यकता है।

आज भी अधिकतर बाल मजदूरों के परिवार में पारिवारिक तनाव है, इसका मुख्य कारण अशिक्षा है। अशिक्षा के चलते अज्ञानता परिवार एवं समाज में व्याप्त है और यही अज्ञानता तनाव में बदल जाता है। गरीबी तथा अशिक्षा के कारण लोगों को योजनाओं की जानकारी नहीं होती है जिससे इनका शोषण किया जाता है। नशे की आदत तथा लापरवाही की वजह से कुछ माता-पिता अपने बच्चों को स्कूल भेजने के बजाय परिवार की आमदनी बढ़ाने के लालच में बाल मजदूरी करने भेज देते हैं। जनसंख्या वृद्धि के कारण गरीबी और अशिक्षा बढ़ रही है जो कि बाल मजदूरी का मुख्य कारण है | जनसंख्या वृद्धि से बेरोजगारी भी बढ़ रही है जिससे बाल-श्रमिकों की रोकथाम में प्रतिकूल प्रभाव पड़ता है।

ग्रामीण क्षेत्रों में अधिकांश बाल श्रमिक कृषि एवं संबद्ध रोजगारों जैसे फसल कटाई, कृषि श्रम, पशुपालन, वानिकी एवं मत्स्य पालन में लगे हैं। कार्यस्थल की अच्छी परस्थितियाँ नहीं होने व जोखिम भरे कार्यों में संलग्रता के कारण बाल श्रमिक स्थाई रूप से अस्वस्थ हो गये हैं। बाल श्रम दूर करने के लिए सबसे जरूरी है गरीबी का उन्मूलन क्योकि गरीबी ही इस समस्या की जड़ है | बच्चों के लिए निःशुल्क और अनिवार्य
प्राथमिक शिक्षा की व्यवस्था करने पर मजदूरी करने वाले बच्चों की संख्या में निश्चित रूप से कमी आ जाएगी | बाल मजदूरी का एक बड़ा कारण सामाजिक चेतना की कमी है।

\section{सुझाव :}

बाल-श्रमिक कृषकों को उनके द्वारा उत्पादित वस्तु का उचित मूल्य दिलाने के लिए सरकार की ओर से पहल करनी चाहिए ताकि किसानों को उचित मूल्य मिल सके और बिचौलिए से किसानों को मुक्ति मिल सके ग्रामीण मजदूर बच्चों को बचत के लिए प्रेरित करने की आवश्यकता है ताकि उनके भविष्य की आवश्यक जरूरतें आसानी से पूरी हो सके | साक्षरता को एक आन्दोलन का रूप देकर उन सभी बाल मज़दूरों को स्कूल से जोड़ा जाय जो अनेक करणों से स्कूल से बाहर होकर एक मजदूर बने हुये है।

\section{संदर्भ ग्रंथ सूची}

1. अवस्थी, दिलीप (1986) ग्लास इण्डस्ट्री : कटिंग कार्नर्स, इण्डिया टुडे, 31 दिसम्बर

2. अवाचट, अनिल (1988) द वार्प ऐण्ड द वेफ्ट-1, इकोनॉमिक ऐण्ड पोलिटिकल वीकली, खण्ड xxxiii, सं. 34, 20 अगस्त,

3. खांडेकर, मंदाकिनी (1970) ए रिपोर्ट ऑन द सिचुएशन ऑव चिल्ड्रेन एण्ड यूथ इनग्रेटर बाम्बे, टाटा इन्स्टीट्यूट ऑव सोशल साइंसेज, मुम्बई

4. खान शमशाद, (1993) "माइग्रैंट चाइल्ड लेबर इन कॉरपेट इण्डस्ट्री ऑव मिर्जापुर-भदोही"

5. गुलाटी, लीला (1981) प्रोफाइल्स इन फीमेल पांवर्टि: ए स्टडी ऑव फाइव पुअर विमेन इन केरल, हिन्दुस्तान पब्लिशिंग कॉपोरेशन, दिल्ली

6. शर्मा सुभाष (2010) बाल—मजदूरी: एक हृष्टिकोण, भारत में बाल-मजदूर 4268-B/3, अंसारी रोड, दरियागंज नयी दिल्ली पृष्ठ संख्या, 15-138

7. सिंह, नूतन (2011) अशिक्षा एवं बालिका मजदूर, बालिका मजदूर, एस. के. पब्लिशिंग कम्पनी, राँची पृष्ठ संख्या, 1-87. 